\title{
Impactos da COVID-19 na saúde mental da população chinesa no início da epidemia: Revisão Integrativa
}

Impacts of COVID-19 on the mental health of the Chinese population at the beginning of the epidemic: Integrative Review Impactos de COVID-19 en la salud mental de la población china al comienzo de la epidemia: revisión integradora

\begin{abstract}
RESUMO
Objetivo: revisar as publicações científicas atuais sobre os impactos da COVID-19 na saúde mental da população chinesa no início da epidemia. Método: Trata-se de uma revisão integrativa com levantamento de estudos em bases de dados online: PubMed, SciELO e LILACS. Os descritores utilizados na busca foram: "COVID 19 AND social isolation" "COVID 19 AND mental health" "COVID 19 AND psychological stress" "COVID 19 AND panic" "COVID 19 AND anxiety" "COVID 19 AND emotions". A busca compreendeu o período de 01 de abril de 2020 a 14 de abril de 2020. Foram selecionados estudos nos idiomas português, inglês e espanhol. Resultados: Quatro atenderam aos critérios de elegibilidade e compuseram a leitura e síntese da presente revisão. Conclusão: Evidenciou-se a presença de indicadores emocionais negativos, como: a ansiedade, depressão, estresse, indignação, diminuição da felicidade, aumento da sensação de risco social e diminuição na satisfação com a vida, redução da qualidade do sono e níveis baixos de capital social. Apesar dos atuais estudos serem realizados apenas em um país, a China, os resultados podem auxiliar no entendimento das condições da saúde mental daquela população e servir de exemplo para a realização de pesquisas em outros países, que devem avaliar a saúde mental da sua própria população com o intuito de direcionar iniciativas de saúde pública.
\end{abstract}

DESCRITORES: Coronavírus; Isolamento Social; Saúde Mental; Ansiedade; Estresse Psicológico.

\section{ABSTRACT}

Objective: to review current scientific publications on the impacts of COVID-19 on the mental health of the Chinese population at the beginning of the epidemic. Method: This is an integrative review with survey of studies in online databases: PubMed, SciELO and LILACS. The descriptors used in the search were: "COVID 19 AND social isolation" "COVID 19 AND mental health" "COVID 19 AND psychological stress" "COVID 19 AND panic" "COVID 19 AND anxiety" "COVID 19 AND emotions". The search covered the period from April 1, 2020 to April 14, 2020. Studies in Portuguese, English and Spanish were selected. Results: Four met the eligibility criteria and composed the reading and synthesis of this review. Conclusion: The presence of negative emotional indicators was evidenced, such as: anxiety, depression, stress, indignation, decreased happiness, increased feeling of social risk and decreased satisfaction with life, reduced sleep quality and low levels of sleep. share capital. Although the current studies are carried out in only one country, China, the results can assist in understanding the mental health conditions of that population and serve as an example for carrying out research in other countries, which should assess the mental health of their own population. in order to direct public health initiatives.

DESCRIPTORS: Coronavirus; Social isolation; Mental Health; Anxiety; Stress, Psychological.

\section{RESUMEN}

Objetivo: revisar las publicaciones científicas actuales sobre los impactos de COVID-19 en la salud mental de la población china al comienzo de la epidemia. Método: Esta es una revisión integradora con una encuesta de estudios en bases de datos en línea: PubMed, SciELO y LILACS. Los descriptores utilizados en la búsqueda fueron: "COVID 19 Y aislamiento social" "COVID 19 Y salud mental" "COVID 19 Y estrés psicológico" "COVID 19 Y pánico" "COVID 19 Y ansiedad" "COVID 19 Y emociones". La búsqueda abarcó el período comprendido entre el 1 de abril de 2020 y el 14 de abril de 2020. Los estudios se seleccionaron en portugués, inglés y español. Resultados: Cuatro cumplieron con los criterios de elegibilidad y compusieron la lectura y síntesis de esta revisión. Conclusión: se evidenció la presencia de indicadores emocionales negativos, tales como: ansiedad, depresión, estrés, indignación, disminución de la felicidad, aumento de la sensación de riesgo social y disminución de la satisfacción con la vida, reducción de la calidad del sueño y bajos niveles de sueño. capital social. Aunque los estudios actuales se llevan a cabo en un solo país, China, los resultados pueden ayudar a comprender las condiciones de salud mental de esa población y servir de ejemplo para llevar a cabo investigaciones en otros países, que deberían evaluar la salud mental de su propia población. para dirigir iniciativas de salud pública. DESCRIPTORES: Coronavirus; Aislamiento Social; Salud Mental; Ansiedad; Estrés psicológico.

RECEBIDO EM: 20/04/2020 APROVADO EM: 21/04/2020 


\section{Miriam Viviane Baron}

Mestra em Promoção da Saúde. Doutoranda no Programa de Pós-Graduação em Medicina e Ciências da Saúde da Pontifícia Universidade Católica do Rio Grande do Sul, PUCRS, Porto Alegre, Brasil. Bolsista de Doutorado da Coordenação de Aperfeiçoamento de Pessoal de Nivel Superior - Brasil (CAPES). https:orcid.org/0000-0002-3673-9750

\section{Ana Julia Palagi Vigano}

Estudante de Medicina na Escola de Medicina da Pontifícia Universidade Católica do Rio Grande do Sul, PUCRS, Porto Alegre, Brasil. https:orcid.org/0000-0002-8591-6418

\section{Gabriela Di Lorenzo Garcia Scherer}

Estudante de Medicina na Escola de Medicina da Pontifícia Universidade Católica do Rio Grande do Sul, PUCRS, Porto Alegre, Brasil. https:orcid.org/0000-0001-5495-8001

\section{Isabella Knorr Velho}

Estudante de Medicina na Escola de Medicina da Pontifícia Universidade Católica do Rio Grande do Sul, PUCRS, Porto Alegre, Brasil. https:orcid.org/0000-0002-7327-8957

\section{Mariana Martins Dantas Santos}

Estudante de Medicina na Escola de Medicina da Pontifícia Universidade Católica do Rio Grande do Sul, PUCRS, Porto Alegre, Brasil. https:orcid.org/0000-0002-4159-9860

\section{Julia Braga da Silveira}

Estudante de Nutrição na Escola de Saúde e Ciências da Vida da Pontifícia Universidade Católica do Rio Grande do Sul, PUCRS, Porto Alegre, Brasil. https:orcid.org/0000-0001-7815-4614

\section{Bartira Ercília Pinheiro da Costa}

Doutora em Biologia Celular e Molecular. Professora Titular Programa de Pós-Graduação em Medicina e Ciências da Saúde. Coordenadora de Pesquisa da Escola de Medicina da Pontifícia Universidade Católica do Rio Grande do Sul, PUCRS, Porto Alegre, Brasil. https:orcid.org/0000-0001-8015-3952

\section{INTRODUÇÃO}

0 SARS-CoV-2 (COVID-19) é um vírus da família coronavírus do tipo beta com fita de RNA simples e, no fim do ano de 2019, foi descoberto como causa de uma série de pneumonias de etiologia desconhecida na cidade de Wuhan, na China ${ }^{(1)}$. Esses pacientes foram epidemiologicamente ligados a um mercado de animais e frutos do mar local. A partir de análises genômicas dos animais vendidos no mercado, verificou-se que o SARS-CoV-2 apresenta $88 \%$ de compatibilidade com dois outros coronavírus do tipo síndrome aguda respiratória severa (SARS) vindos de morcegos; assim, considerou-se que a origem do SARS-CoV-2 seria zoonótica ${ }^{(2)}$.

O SARS-CoV-2 apresenta uma taxa de transmissão maior do que o SARS-CoV, responsável por um surto em 2002, devido, provavelmente, a uma recombinação genética numa proteína $S$ na região $R B D$ do SARS-CoV-2 que aprimorou sua ca- pacidade de transmissão ${ }^{(1)}$. A transmissão entre humanos ocorre principalmente via contato direto ou através de gotículas e aerossóis oriundos de tosses e espirros de indivíduos infectados ${ }^{(2)}$. Devido à alta taxa de transmissão do SARS-CoV-2, em 30 de janeiro a Organização Mundial da Saúde (OMS) declarou que o surto de COVID-19 é uma Emergência de Saúde Pública de Interesse Internacional e, no dia 11 de fevereiro, foi declarada, pela OMS, como uma pandemia.

Emergências em saúde pública como o surgimento repentino da epidemia da COVID-19, a velocidade de transmissão rápida, o poder infeccioso do vírus e a necessidade de distanciamento social inevitavelmente causam incertezas e não apenas ameaçam a saúde física das pessoas, mas também a saúde mental ${ }^{(3)}$. Especialmente em termos de emoções negativas em que é possível que as pessoas desenvolvam (por exemplo, estresse, ansiedade, indignação e depressão) e em termos de avaliação cognitiva negativa (por exemplo, aumento da sensibilidade a riscos sociais e diminuição da felicidade e de satisfação com a vida) podendo chegar a extremos como o delírio, psicose e suicídio ${ }^{(4-9)}$.

O indivíduo estressado apresenta respostas fisiológicas devido a alterações do sistema nervoso autônomo e endócrino(3). Ocorre aumento da liberação de hormônios, como o cortisol e prolactina, na corrente sanguínea que produz manifestações, como: a aceleração dos batimentos cardíacos, aumento da pressão arterial, palpitações, aumento do índice glicêmico no sangue, distúrbio de apetite, problemas gastrointestinais, distúrbio de sono e humor, cefaleia, dores no corpo, alterações endócrinas, entre outras ${ }^{(3,10)}$. Além disso, a ação desses hormônios sobre as interleucinas reduz a capacidade de proliferação e ação dos leucócitos, causando supressão do sistema imunológico ${ }^{(11)}$, que pode deixar o corpo predisposto a infecções ${ }^{(12)}$.

De acordo com a OMS, saúde mental é o sentir-se bem consigo e com os outros ao seu redor, saber lidar com 
emoções, ter estratégias de vida como crescimento, desenvolvimento e autorrealização, buscando sempre a plenitude para viver e respeitando o limite do outro $^{(13)}$. Mas quando não controladas, emoções e sentimentos podem se tornar problemas sérios de saúde, causando alterações fisiológicas que interferem em sistemas corporais e que podem deixar o corpo predisposto a doenças ${ }^{(12)}$, além de agravar para doenças como a depressão e ansiedade ${ }^{(13)}$.

\begin{tabular}{l} 
Quadro 1. Etapas para a revisão integrativa. Porto Alegre, RS, Brasil, 2020 \\
ETAPAS PARA A REVISÃo \\
\hline - Identificação do problema de pesquisa; \\
\hline - Definição do objetivo; \\
\hline - Seleção da amostra e critérios de elegibilidade; \\
\hline 4 - Definição das informações a serem extraídas dos artigos; \\
\hline 5 - Análise dos estudos incluídos na revisão; \\
\hline 6 - Interpretação de resultados; \\
\hline - Síntese do conhecimento.
\end{tabular}

Quadro 2. Grau de evidência dos estudos incluídos na revisão. Porto Alegre, RS, Brasil, 2020

\begin{tabular}{|l|l|}
\hline $\begin{array}{l}\text { GRAU DE } \\
\text { EVIDÊNCIA }\end{array}$ & FONTE DA EVIDÊNCIA \\
\hline Grau 1A & $\begin{array}{l}\text { Evidências resultantes de revisões sistemáticas e metanálises de } \\
\text { ensaios clínicos comparáveis. Estudos controlados randomizados bem } \\
\text { delineados com desfecho clínico relevante. }\end{array}$ \\
\hline Grau 1B & $\begin{array}{l}\text { Evidências baseadas em estudos controlados randomizados com estrei- } \\
\text { to intervalo de confiança. }\end{array}$ \\
\hline Grau 1C & $\begin{array}{l}\text { Evidências de resultados do tipo "tudo ou nada". Estudo de série de } \\
\text { casos controlados. }\end{array}$ \\
\hline Grau 2A & $\begin{array}{l}\text { Evidências resultantes de revisão sistemática homogênea de estudos } \\
\text { de coorte (com grupos de comparação e controle de variáveis). }\end{array}$ \\
\hline Grau 2B & $\begin{array}{l}\text { Evidências baseadas em estudos de coorte com pobre qualidade de } \\
\text { randomização, controle ou sem acompanhamento longo, estudo de } \\
\text { coorte transversal. }\end{array}$ \\
\hline Grau 2C & $\begin{array}{l}\text { Evidências de resultados de pesquisas (observação de resultados tera- } \\
\text { pêuticos ou evolução clínica). }\end{array}$ \\
\hline Grau 3A & $\begin{array}{l}\text { Evidências resultantes de revisão sistemática homogênea de estudos } \\
\text { de caso com grupo-controle. }\end{array}$ \\
\hline Grau 3B & Evidências baseadas em estudos de caso com grupo-controle. \\
\hline Grau 4 & $\begin{array}{l}\text { Evidências provenientes de relatos de caso e série sem definição de } \\
\text { caso-controle. }\end{array}$ \\
\hline Grau 5 & $\begin{array}{l}\text { Evidências provenientes da opinião de autoridades ou especialistas. } \\
\text { Revisão da literatura não-sistemática. }\end{array}$ \\
\hline
\end{tabular}

mental da população chinesa no início da epidemia? Assim, este estudo teve como objetivo revisar as publicações científicas atuais sobre os impactos da COVID-19 na saúde mental da população chinesa no início da epidemia.

\section{METODOLOGIA}

Trata-se de uma revisão integrativa de estudos. Para a preparação desta revisão, foram seguidas as etapas apresentadas no Quadro 1, abaixo:

Foram incluídos estudos que abordassem o tema COVID-19 e o impacto na saúde mental da população chinesa no início da epidemia, sem limite de idade. Os critérios de exclusão foram cartas ao editor, comentários, comunicações breves, relatos de caso e capítulos de livros.

Foram pesquisadas três bases de dados como fontes de levantamento dos estudos: Public Medline (PubMed), Scientific Electronic Library Online (SciELO) e Literatura Latino-Americana e do Caribe em Ciências da Saúde (LILACS). Os descritores utilizados foram: "COVID 19 AND social isolation" "COVID 19 AND mental health" "COVID 19 AND psychological stress" "COVID 19 AND panic" "COVID 19 AND anxiety" "COVID 19 AND emotions". A busca compreendeu o período de 01 de abril de 2020 a 14 de abril de 2020. Foram selecionados estudos disponíveis nos idiomas português, inglês e espanhol.

Após a busca completa, os títulos e resumos dos estudos foram lidos e avaliados por dois revisores de forma independente. Após a seleção por título e resumo, os revisores avaliaram os artigos por completo. As discordâncias entre os revisores foram resolvidas por consenso. A extração dos dados foi realizada por meio de um formulário padronizado.

A análise do nível de evidência, segundo o tipo de estudo, seguiu a classificação de evidências proposta pelo Oxford Centre Evidence-Based Medicine ${ }^{(14)}$ também utilizada por pesquisadores em revisão integrativa pregressa ${ }^{(15)}$. A classificação das evidências está descrita no Quadro 2. 


\section{artigo}

Para organizar e sumariar os principais achados dos estudos e compor o banco de dados considerou-se os seguintes tópicos: autor/ano, tipo de estudo, amostra (sujei- tos)/ características, objetivo, medidas de avaliação da saúde mental, principais resultados e nível de evidência. A síntese do conhecimento foi apresentada de forma

\section{Figura 1. Fluxograma do estudo. Porto Alegre, RS, Brasil, 2020}

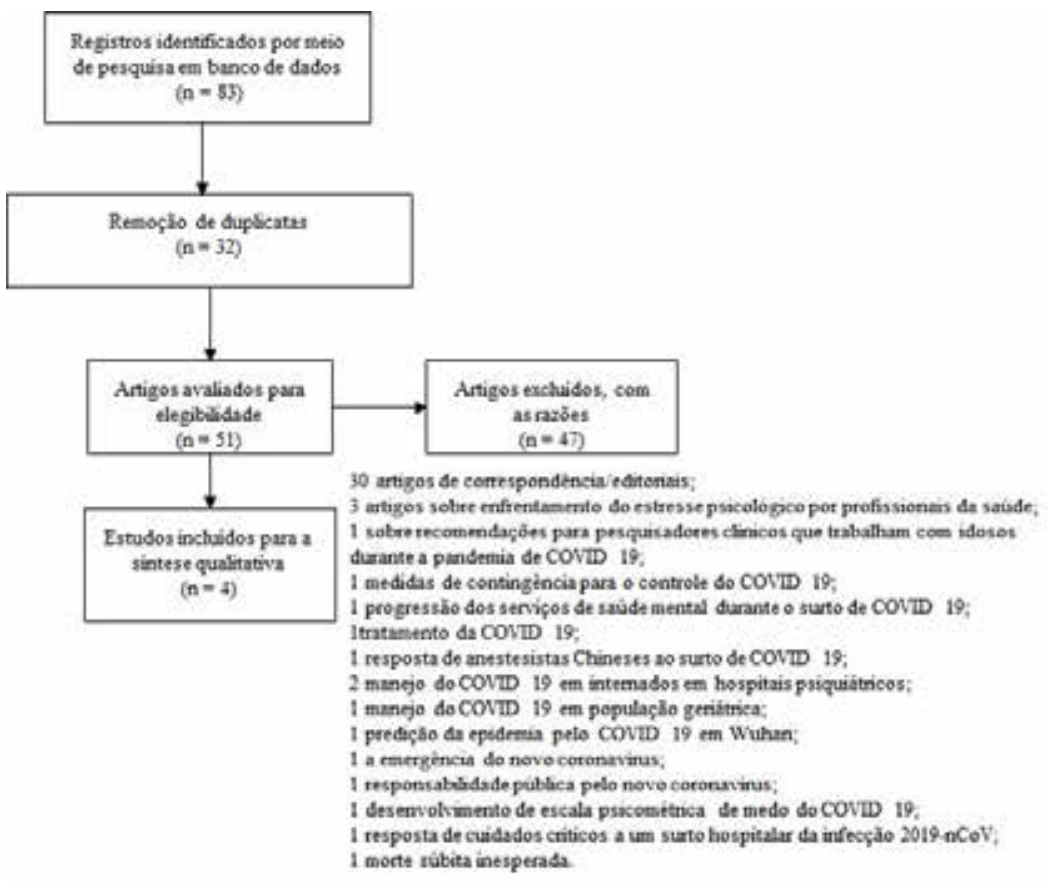

Quadro 3. Síntese dos estudos selecionados. Porto Alegre, RS, Brasil, 2020

\begin{tabular}{|c|c|c|c|c|c|c|}
\hline $\begin{array}{l}\text { AUTOR/ } \\
\text { ANO }\end{array}$ & $\begin{array}{l}\text { TIPO DE } \\
\text { ESTUDO }\end{array}$ & $\begin{array}{c}\text { AMOSTRA/ } \\
\text { CARACTERÍSTICAS }\end{array}$ & OBJETIVO & $\begin{array}{l}\text { MEDIDAS DE AVALIAÇÃO } \\
\text { DA SAÚDE MENTAL }\end{array}$ & PRINCIPAIS RESULTADOS & NE \\
\hline $\begin{array}{l}\text { Xiao, } \\
\text { Zhang, } \\
\text { Kong, } \\
\text { Li, Yang, } \\
2020 \text { [16] }\end{array}$ & $\begin{array}{l}\text { Estudo } \\
\text { transvesal }\end{array}$ & $\begin{array}{l}170 \text { pessoas (59,4\% } \\
\text { do sexo masculino) } \\
\text { com idade média de } \\
37,7 \pm 4,1 \text { anos e autoi- } \\
\text { soladas em casa por } 14 \\
\text { dias na China, devido à } \\
\text { epidemia da COVID-19 } \\
\text { em janeiro de } 2020 . \\
\text { Indivíduos adultos que } \\
\text { estavam com sinais } \\
\text { leves da covID-19, } \\
\text { pessoas em contato } \\
\text { próximo com pacientes } \\
\text { infectados, casos sus- } \\
\text { peitos e pessoas que } \\
\text { podem ter sido expos- } \\
\text { tas ao vírus pelo am- } \\
\text { biente completaram os } \\
\text { questionários no tercei- } \\
\text { ro dia de isolamento. }\end{array}$ & $\begin{array}{l}\text { Investigar os } \\
\text { efeitos do ca- } \\
\text { pi-tal social } \\
\text { na qualidade } \\
\text { do sono e os } \\
\text { mecanismos } \\
\text { envolvidos em } \\
\text { pessoas que } \\
\text { se isolaram em } \\
\text { casa por } 14 \text { dias } \\
\text { em janeiro de } \\
2020 \text { durante } \\
\text { epidemia de } \\
\text { COVID-19. }\end{array}$ & $\begin{array}{l}\text { Foram coletados dados } \\
\text { demográficos, socio- } \\
\text { lógicos e informações } \\
\text { relacionadas à doença. } \\
\text { Foram utilizados } 4 \text { ques- } \\
\text { tionários: PSCI - } 16 \text { para } \\
\text { avaliar o capital social in- } \\
\text { dividual, SAS para medir } \\
\text { os níveis de ansiedade, } \\
\text { SASR para medir o es- } \\
\text { tresse e PSQI para medir } \\
\text { a qualidade do sono. }\end{array}$ & $\begin{array}{l}\text { Durante o período de au- } \\
\text { toisolamento individual devi- } \\
\text { do à epidemia da COVID-19 } \\
\text { na China observou-se que } \\
\text { níveis baixos de capital } \\
\text { social foram associados a } \\
\text { niveis aumentados de an- } \\
\text { siedade e estresse, mas } \\
\text { níveis aumentados de } \\
\text { capital social foram po- } \\
\text { sitivamente associados } \\
\text { ao aumento da qualidade } \\
\text { do sono. A ansieda-de foi } \\
\text { associada ao estresse e à } \\
\text { redução da qualidade do } \\
\text { sono, e a combinação de } \\
\text { ansiedade e estresse re- } \\
\text { duziu os efeitos positivos } \\
\text { do capital social na quali- } \\
\text { dade do sono. }\end{array}$ & $2 B$ \\
\hline
\end{tabular}




\begin{tabular}{|c|c|c|c|c|c|c|}
\hline $\begin{array}{l}\text { Li, Wang, } \\
\text { Xue, Zhao, } \\
\text { Zhu, } 2020 \\
{[17]}\end{array}$ & $\begin{array}{l}\text { Coleta em } \\
\text { base de } \\
\text { dados do } \\
\text { app Weibo }\end{array}$ & $\begin{array}{l}\text { Foram amostradas e } \\
\text { avaliadas as atividades } \\
\text { de } 17.865 \text { usuários do } \\
\text { app Weibo (mediana } \\
\text { de idade de } 33 \text { anos e } \\
25,2 \% \text { eram homens) } \\
\text { entre } 13 \text { e } 26 \text { de janei- } \\
\text { ro de } 2020.0 \text { anúncio } \\
\text { da epidemia se deu no } \\
\text { dia } 20 \text { de janeiro de } \\
2020 \text { na China, e com- } \\
\text { pararam-se dados dos } \\
\text { usuários nos dias an- } \\
\text { teriores e posteriores } \\
\text { ao anúncio. Foram co- } \\
\text { letadas (1) informações } \\
\text { do perfil do usuário, (2) } \\
\text { comportamentos de } \\
\text { rede e (3) mensagens } \\
\text { do Weibo. Os critérios } \\
\text { de inclusão foram (1) } \\
\text { publicar pelo menos } \\
50 \text { posts originais na } \\
\text { plataforma no período } \\
\text { de } 31 \text { de dezembro de } \\
2019 \text { a } 26 \text { de janeiro } \\
\text { de } 2020, \text { (2) o perfil não } \\
\text { ser institucional e (3) a } \\
\text { região do usuário de- } \\
\text { clarada ser China. }\end{array}$ & 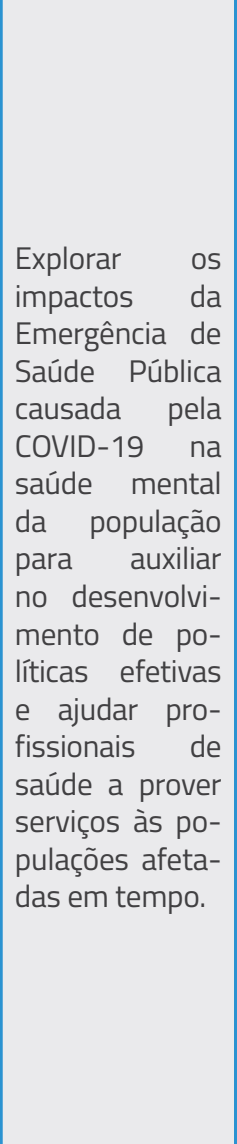 & $\begin{array}{l}\text { Foi utilizado o Online Eco- } \\
\text { logical Recognition (OER), } \\
\text { que faz o reconhecimen- } \\
\text { to automático do perfil } \\
\text { psicológico utilizando } \\
\text { modelos preditivos de } \\
\text { aprendizado de máqui- } \\
\text { na. Para extrair recursos } \\
\text { de conteúdo foi utilizado } \\
\text { o sistema Text Mind do } \\
\text { LPCCIPACC. Por meio do } \\
\text { Text Mind foi feita uma } \\
\text { separação das palavras } \\
\text { de acordo com sua ca- } \\
\text { tegoria gramatical e, } \\
\text { então, com o dicionário } \\
\text { LIWC chinês, essas pa- } \\
\text { lavras foram separadas } \\
\text { em categorias psicoló- } \\
\text { gicas. Foram calculadas } \\
\text { frequência de palavras, } \\
\text { pontuação de indicadores } \\
\text { emocionais negativos e } \\
\text { positivos e indicadores } \\
\text { cognitivos, e, compara- } \\
\text { das as diferenças das ca- } \\
\text { racterísticas psicológicas } \\
\text { antes e depois da decla- } \\
\text { ração da epidemia. }\end{array}$ & $\begin{array}{l}\text { Após } 20 \text { de Janeiro, indi- } \\
\text { cadores emocionais de } \\
\text { traços psicológicos nega- } \\
\text { tivos aumentaram como: } \\
\text { ansiedade, depressão e } \\
\text { indignação; enquanto in- } \\
\text { dicadores emocionais de } \\
\text { traços psicológicos posi- } \\
\text { tivos diminuíram, como } \\
\text { a escala de felicidade de } \\
\text { Oxford e satisfação com a } \\
\text { vida. Os resultados podem } \\
\text { fornecer referências efeti- } \\
\text { vas aos formuladores de } \\
\text { políticas e profissionais de } \\
\text { saúde no atendimento da } \\
\text { população afetada. }\end{array}$ & $2 B$ \\
\hline $\begin{array}{l}\text { Wang, } \\
\text { Pan, Wan, } \\
\text { Tan, Xu, } \\
\text { Ho, Ho, } \\
2020[6]\end{array}$ & $\begin{array}{l}\text { Estudo } \\
\text { transvesal }\end{array}$ & $\begin{array}{l}\text { Foi feito uma pes- } \\
\text { quisa online e anôni- } \\
\text { ma por amostragem } \\
\text { de bolas de neve nos } \\
\text { dias } 31 / 01 / 2020 \text { a } \\
02 / 02 / 2020 \text { por inter- } \\
\text { médio da plataforma } \\
\text { SurveyStar (China), que } \\
\text { foi enviado para estu- } \\
\text { dantes universitários, e } \\
\text { estes foram encoraja- } \\
\text { dos a enviar para outras } \\
\text { pessoas. Obteve-se } \\
\text { resposta de } 1210 \text { entre- } \\
\text { vistados de } 194 \text { cidades } \\
\text { da China. Dos pesquisa- } \\
\text { dos, } 53,1 \% \text { tinham entre } \\
21,4 a 30,8 \text { anos e } 67,4 \% \\
\text { eram mulheres. }\end{array}$ & $\begin{array}{l}\text { O objetivo do } \\
\text { estudo foi pes- } \\
\text { quisar a popu- } \\
\text { lação chinesa } \\
\text { para compreen- } \\
\text { der os níveis de } \\
\text { impacto psico- } \\
\text { lógico, ansieda- } \\
\text { de, depressão } \\
\text { e estresse du- } \\
\text { rante o estágio } \\
\text { inicial do surto } \\
\text { de COVID-19. }\end{array}$ & $\begin{array}{l}\text { O questionário continha } \\
\text { questões que cobriam } \\
\text { as áreas: (1) dados de- } \\
\text { mográficos, (2) sintomas } \\
\text { físicos nos últimos } 14 \\
\text { dias, (3) história de con- } \\
\text { tato com a COVID-19, } \\
\text { (4) conhecimentos e } \\
\text { preocupações com a } \\
\text { COVID-19, (5) medidas } \\
\text { de precaução contra a } \\
\text { COVID-19, (6) informa- } \\
\text { ções adicionais sobre a } \\
\text { COVID-19, (7) impacto } \\
\text { psicológico do surto da } \\
\text { COVID-19 e (8) estado de } \\
\text { saúde mental. O impacto } \\
\text { psicológico foi avaliado } \\
\text { pela escala IESR e o es- } \\
\text { tado da saúde mental } \\
\text { foi avaliado pela escala } \\
\text { DASS-21. }\end{array}$ & $\begin{array}{l}\text { Mais da metade dos en- } \\
\text { trevistados classificou seu } \\
\text { impacto psicológico como } \\
\text { moderado a grave e cerca } \\
\text { de um terço relatou an- } \\
\text { siedade moderada a gra- } \\
\text { ve. Sexo feminino, status } \\
\text { de estudante e sintomas } \\
\text { físicos específicos foram } \\
\text { associados a um maior } \\
\text { impacto psicológico do } \\
\text { surto e a níveis mais altos } \\
\text { de estresse, ansiedade e } \\
\text { depressão. Informações } \\
\text { específicas de saúde atua- } \\
\text { lizadas e precisas e certas } \\
\text { medidas de precaução fo- } \\
\text { ram associadas a um me- } \\
\text { nor impacto psicológico do } \\
\text { surto e a níveis mais bai- } \\
\text { xos de estresse, ansiedade } \\
\text { e depressão. }\end{array}$ & $2 B$ \\
\hline
\end{tabular}




\begin{tabular}{|c|c|c|c|c|c|c|}
\hline $\begin{array}{l}\text { Wang, Di, } \\
\text { Ye, Wei, } \\
2020 \text { [3] }\end{array}$ & $\begin{array}{l}\text { Estudo } \\
\text { transvesal }\end{array}$ & $\begin{array}{l}\text { Pesquisa do estado psi- } \\
\text { cológico da população } \\
\text { de algumas regiões na } \\
\text { China no período de } 6 \text { a } \\
9 \text { de fevereiro de } 2020 \text {. } \\
\text { Foram avaliados } 600 \\
\text { questionários. A idade } \\
\text { média dos pesquisa- } \\
\text { dos era } 34 \pm 12 \text { anos e } \\
55,5 \% \text { eram mulheres. } \\
\text { Critérios de inclusão (1) } \\
\text { ter } 18 \text { anos ou mais (2) } \\
\text { completar todo o ques- } \\
\text { tionário. }\end{array}$ & $\begin{array}{l}\text { Investigar o es- } \\
\text { tado psicológico } \\
\text { público e fato- } \\
\text { res relacionados } \\
\text { durante o surto } \\
\text { da COVID-19. }\end{array}$ & $\begin{array}{l}\text { O questionário incluiu: (1) } \\
\text { Dados sociodemográfi- } \\
\text { cos; (2) SAS - para avaliar } \\
\text { os sentimentos subjeti- } \\
\text { vos de ansiedade; (3) SDS } \\
\text { - para medir o grau de de- } \\
\text { pressão. }\end{array}$ & $\begin{array}{l}\text { Os } 600 \text { participantes foram } \\
\text { considerados psicologica- } \\
\text { mente estáveis. As taxas } \\
\text { de não ansiedade e não } \\
\text { depressão foram de } 93,6 \% \\
\text { e } 82,8 \% \text {, respectivamente. } \\
\text { Houve ansiedade em } 6,3 \% \\
\text { e depressão em } 17,7 \% \text { dos } \\
\text { participantes da pesquisa. }\end{array}$ & $2 \mathrm{~B}$ \\
\hline
\end{tabular}

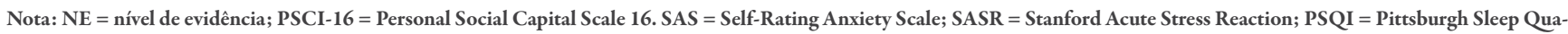

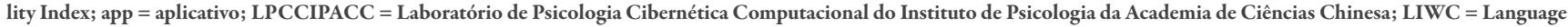
Inquiry and Word Count; IES-R = Impact of Event Scale-Revised; DASS-21 = Depression, Anxiety and Stress Scale; SDS = Self-Rating Depression Scale.

fação com a vida ${ }^{(17)}$, redução da qualidade do sono e níveis baixos de capital social ${ }^{(16)} \mathrm{em}$ avaliação da população no início da epidemia da COVID-19 na China, entre janeiro e fevereiro de 2020.

Os quatro estudos avaliados mostram grau de evidência (2B), apresentando evidências e resultados robustos que podem fornecer recomendações aos formuladores de políticas públicas para planejar e lutar contra a COVID-19, preparar as equipes prestadoras de saúde com evidências científicas e melhorar os sentimentos da população.

\section{Resultados de avaliação da saúde mental}

Estudo avaliou os sentimentos subjetivos de ansiedade por intermédio da escala SAS e o grau de depressão com a escala SDS no início da epidemia na China. Os resultados dos 600 questionários do estudo mostraram que, durante o surto de COVID-19, 562 pessoas $(93,6 \%)$ não apresentaram sintomas de ansiedade e 38 pessoas (6,3\%) sentiram ansiedade; 497 pessoas $(82,8 \%)$ não apresentavam sintomas de depressão e 103 pessoas $(17,1 \%)$ estavam deprimidas. A análise de correlação para os escores SAS e SDS mostrou-se positiva $(r=0,696 ; \mathrm{p}<0,001)$, evidenciando que as pessoas com ansiedade eram propensas à depressão e as pessoas deprimidas apresentavam tendência a estarem ansiosas. A pesquisa também mostrou que pessoas com 40 anos ou menos tinham um risco aumentado de ansiedade em relação aquelas com mais de 40 anos, e as mulheres apresentavam maior risco de ansiedade do que os homens ${ }^{(3)}$.

Os autores constataram que a capacidade de autorregulação psicológica e a qualidade psicológica aumentam com o aumento da idade. As mulheres, além de terem seus papéis profissionais, assumem o papel social de cuidador principal da família e são mais delicadas e sensíveis do que os homens, e, portanto, as mulheres são mais propensas à ansiedade $^{(3)}$ e também à depressão ${ }^{(18)}$.

Pesquisadores também avaliaram a depressão, ansiedade e estresse por meio da escala DASS-21 e o impacto psicológico da COVID-19 na população chinesa no início da epidemia por intermédio da escala IES-R. Dos pesquisados, 53,8\% classificou o impacto psicológico do surto como moderado ou grave; $16,5 \%$ relataram sintomas depressivos moderados a graves; $28,8 \%$ relataram sintomas de ansiedade moderados a graves; e 8,1\% relataram níveis de estresse moderado a grave. A maioria dos pesquisados passou de 20 a 24 horas por dia em casa $(84,7 \%)$; sentiam-se preocupados com os familiares contraírem COVID-19 (75,2\%); e ficaram satisfeitos com a quantidade de informações de saúde disponíveis $(75,1 \%)$. No entanto, ser do sexo feminino, ter status de estudante, ter sintomas físicos específicos (mialgia, tontura, coriza) e mau estado de saúde autoavaliado foram significativamente associados a um maior impacto psicológico do surto e níveis mais altos de estresse, ansiedade e depressão $(\mathrm{p}<0,05)$. Da mesma forma, ter informações de saúde precisas e atualizadas (tratamento, situação lo- cal do surto) e medidas de precaução (higiene das mãos, uso de máscara) estiveram associadas a um menor impacto psicológico do surto e a níveis mais baixos de estresse, ansiedade e depressão - $\mathrm{p}<0,05^{(6)}$.

Estes achados reforçam que as autoridades de saúde devem identificar grupos de risco (como mulheres, estudantes) com base em informações sociodemográficas para intervenções psicológicas precoces, visto que estes sofrem com um maior impacto psicológico do surto, além de apresentar níveis mais altos de estresse, ansiedade e depressão ${ }^{(6)}$. Esse achado corrobora com estudo epidemiológico pregresso que observou que as mulheres estão em maior risco de depressão que os homens ${ }^{(6,18)}$.

Outro estudo investigou os efeitos do capital social na qualidade do sono e os mecanismos envolvidos em pessoas que se isolaram em casa por 14 dias no início da epidemia da COVID-19. O capital social individual foi avaliado pelo questionário PSCI-16; a ansiedade pela escala SAS; o estresse foi avaliado usando o questionário SASR; e o sono por intermédio do questionário PSQI. As análises mostraram que PSCI-16 para capital social estava associado positivamente ao SAS para ansiedade $(\mathrm{r}=0,619 ; \mathrm{p}<0,01)$, ao SARS para estresse $(r=0,543 ; \mathrm{p}<0,01)$ e ao PSQI para a qualidade do sono $(r=0,479 ; \mathrm{p}<0,01)$. O escore SAS para ansiedade dos participantes foi associado positivamente ao escore SASR para estresse $(r=0,553 ; \mathrm{p}<0,01)$ e ao PSQI para qualidade do sono $(r=0,523 ; p<0,01)$. $O$ escore SASR para estresse foi positivamente 
associado ao escore PSQI para qualidade do sono $(r=0,628 ; p<0,01)$. Os achados mostraram que níveis baixos de capital social foram associados a níveis aumentados de ansiedade e estresse, mas níveis aumentados de capital social foram positivamente associados ao aumento da qualidade do sono. Por outro lado, a ansiedade foi associada ao estresse e à redução da qualidade do sono, e a combinação de ansiedade e estresse reduziu os efeitos positivos do capital social na qualidade do sono ${ }^{(16)}$.

O capital social refere-se à confiança social, pertencimento e participação social, esses recursos estão associados a uma rede duradoura de reconhecimento mútuo ${ }^{(19)}$. Quando indivíduos são isolados, como no caso de epidemias, grupos sociais em redes sociais on-line podem oferecer apoio mútuo e reduzir a ansiedade causada pelo isolamento ${ }^{(20)}$ e, consequentemente, o estresse, que pode melhorar a qualidade do sono ${ }^{(16)}$.

Uma pesquisa buscou explorar os impactos da COVID-19 na saúde mental das pessoas e ajudar os formuladores de políticas públicas a desenvolver artifícios incorporáveis e ajudar os profissionais de saúde a fornecer serviços oportunos à população. Para tal, os pesquisadores analisaram as postagens do Weibo (aplicativo de rede social) de 17.865 usuários ativos por intermédio de um programa que faz o reconhecimento automático do perfil psicológico - OER - e utiliza modelos preditivos baseados em aprendizado de máquina. Com auxílio da ferramenta Text Mind, foi feita uma separação das palavras mais usadas nas postagens do Weibo de acordo com sua categoria gramatical e, então, com o dicionário chinês Language Inquiry and Word Count (LIWC), essas palavras foram separadas em categorias psicológicas. Foram calculados a frequência das palavras, escores de indicadores emocionais (ansiedade, depressão, indignação e felicidade) e indicadores cognitivos (sensação de risco social e satisfação com a vida) a partir dos dados coletados, e comparados às diferenças das características psicológicas antes e após da declaração da epidemia no dia 20 de janeiro. Os resultados mostraram que após 20 de janeiro, indicadores emocionais negativos aumentaram em ansiedade $(\mathrm{t}(17.747)=$ $-35.962, \mathrm{p}<0,001)$, depressão $(\mathrm{t}(17.747)=$
-10.717, p <0.001) e indignação ( $t(17.747)$ $=5.500, p<0,001)$, enquanto indicadores emocionais positivos diminuíram na escala de felicidade de Oxford $(t(17.747)=3.120$, $\mathrm{p}<0,01)$. Também houve aumento de indicadores cognitivos de sensação de risco $\operatorname{social}(t(17.747)=3.120, p<0,01)$, e diminuição na satisfação com a vida $(\mathrm{t}(17.747)=$ $5.500, p<0,001)$. O estudo mostrou que as emoções negativas (por exemplo, ansiedade, depressão e indignação) e a sensibilidade aos riscos sociais aumentou, enquanto as pontuações de emoções positivas (por exemplo, felicidade de Oxford) e satisfação com a vida diminuíram. Da mesma forma, o estudo evidenciou que as pessoas estavam mais preocupadas com a sua saúde e da família, e menos preocupadas com o lazer e amigos ${ }^{(17)}$.

Mudanças psicológicas causadas por emergências de saúde pública podem ser refletidas diretamente nas emoções e na cognição de indivíduos $^{(21)}$ e as mudanças psicológicas podem ser monitoradas em determinado tempo por meio de indicadores emocionais (por exemplo, emoções positivas e negativas) e cognitivos (por exemplo, sensação de risco social e satisfação com a vida). O uso de dados de mídia social pode fornecer uma compreensão oportuna de impacto de emergências de saúde pública na saúde mental dos indivíduos durante um período de epidemia ${ }^{(17)}$.

\section{Recomendações para gestores de polí- ticas públicas e profissionais da saúde}

Em períodos de emergência de saúde pública, como no caso da epidemia da COVID-19, profissionais da saúde e Governo devem prestar atenção especial à saúde mental do público e buscar oferecer cuidados psicológicos apropriados e fortalecer o capital social ${ }^{(3,16)}$. No intuito de identificar as necessidades psicológicas e dar suporte à população que apresenta ou não os sintomas da COVID-19, o uso de smartphones e da mídia social tornaram-se ferramentas importantes de difusão de informações aliadas à promoção da saúde. De acordo com pesquisadores, o uso de dados de mídias sociais e pesquisas on-line podem promover o entendimento oportuno do impacto das emergências de saúde pública na saúde mental dos indivíduos durante o período de epidemia ${ }^{(6,17)}$.
Além disso, para os responsáveis pelas políticas públicas, sugere-se:

- Desenvolver uma política consistente com procedimentos para reportar sobre a transmissão disseminada da doença, os casos confirmados e número de mortos atualizados, assim como outros dados da situação epidêmica. Pois as pessoas precisam entender as informações corretamente para evitar respostas excessivas de estresse causadas por interpretação equivocada ${ }^{(6,17)}$. Agências locais devem fornecer informações em linguagem informal para auxiliar pessoas com baixo nível de conhecimento com esclarecimentos pertinentes sobre a epidemia da COVID-19; recomenda-se que façam isso junto com o governo e autoridades de saúde para certificar a autenticidade das informações e reduzir o impacto dos rumores sobre $\mathrm{o}$ assunto ${ }^{(6)}$.

- Expandir o conhecimento e consciência da população sobre o progresso contínuo nas medidas decisórias. $\mathrm{O}$ maior envolvimento e conhecimento do público diminui a indignação frente às falhas em controlar a epidemia ${ }^{(17)}$. A equipe médica profissional deve fornecer educação em saúde on-line para reduzir a incerteza e o pânico causados pela falta de conhecimento sobre o novo coronavírus. Além disso, as autoridades de saúde devem fornecer máscaras, sabonetes, álcool e outros produtos de higiene à populaçãa ${ }^{(6,16)}$.

- Garantir o serviço de tratamento médico e informar a população sobre como acessá-lo convenientemente, pois quando as pessoas se sentem mais no controle sobre os riscos envolvidos, isso evita o excesso de sensação de risco social ${ }^{(17)}$. Deve-se identificar as necessidades psicológicas da população que apresenta sintomas físicos durante a epidemia. Fornecer recursos para suporte psicológico e intervenções em pessoas com sintomas de COVID-19, principalmente durante a hospitalização( ${ }^{(6)}$. Assim como, assistentes sociais e psicoterapeutas podem fornecer ajuda por meio de chat em aplicativos, portais on-line ou suporte por telefone, e incentivar a comunica- 
ção com parentes e amigos com o uso da internet ou telefone ${ }^{(6,16)}$. Essas ações podem auxiliar na sensação de estabilidade e aliviar a ansiedade e a depressão ${ }^{(3,17)}$.

- Prover acesso a entretenimento doméstico para garantir boa qualidade de vida ${ }^{(17)}$.

\section{Recomendações para a população}

Pesquisadores sugerem que a população deve prestar atenção às informações que a mídia social apresenta e evitar absorver informações enganosas que, inevitavelmente, podem levar a reações adversas, como o pânico e a depressão. Também recomendam que a sociedade deva fortalecer a publicidade de prevenção e controle de epidemias e educar o público, fornecendo exemplos específicos de métodos científicos de prevenção, como a lavagem adequada das mãos, o uso de máscaras e as precauções adequadas para implementar quando sair de casa, por exemplo ${ }^{(3,6)}$.

Além disso, o público deve fazer uso de serviços oferecidos pelo governo e profissionais da saúde (por exemplo, psiquiatras, psicólogos, enfermeiros e assistentes sociais) por intermédio de plataforma on-line, aplicativos, chats ou ligações telefônicas sempre que houver necessidade de aconselhamento psicológico, esclarecimentos sobre a doença e a epidemia, entre outros; o que pode ajudar a aliviar os distúrbios e danos psicológicos causados pela epidemia e fortalecer o capital social ${ }^{(3,16)}$.

Pesquisadores recomendam que o público mantenha uma rotina diária. Que neste momento, as pessoas busquem relaxar e aproveitar o tempo livre, enquanto outras aproveitam a oportunidade para estudar e descansar ${ }^{(3)}$. Da mesma maneira, o uso de terapia comportamental pode focar em exercícios de relaxamento que contrariem a ansiedade e em uma rotina de atividades domésticas que contrariem a depressão. A acupressão e técnicas de liberdade emocional baseadas na medicina tradicional chinesa podem beneficiar a saúde mental durante a epidemia da COVID-19(6).

\section{Limitações}

As limitações referem-se aos estudos todos terem sido realizados na China e, portanto, não refletem os resultados $\mathrm{da}$ população de outros países que pode ser influenciada por questões culturais, de clima, de temperatura, sociais, econômicas, políticas entre outras. Além disso, os estudos envolveram indivíduos com idade entre $8 \mathrm{e}$ 72 anos, e duas pesquisas ${ }^{(16,17)}$ não avaliaram a saúde mental de subgrupos, como jovens, adultos e idosos que pode ser diferente nos grupos etários, como mostra um estudo ${ }^{(3)}$ em que pessoas com 40 anos ou menos tinham um risco aumentado de ansiedade quando comparadas com aquelas de mais de 40 anos, durante o surto da COVID-19 em algumas regiôes da China.

Por outro lado, em um estudo houve uma amostragem excessiva de um grupo específco de participantes (estudantes), levando a respostas com viés de seleção; em que os resultados não podem ser extrapolados para a população em geral, especialmente para pessoas com níveis escolares menores ${ }^{(6)}$. Foram adotados questionários on-line, devido aos poucos recursos disponíveis durante o surto da COVID-19; e estudos com amostragem não aleatória, o que pode trazer informações tendenciosas, abre a possibilidade da pesquisa não atingir regiões subdesenvolvidas devido à disponibilidade limitada de tecnologia, e pode omitir pessoas que não se sintam confortáveis em usar a tecnologia e a Internet e, portanto, não representa os dados da população geral da China ${ }^{(3,6)}$.

Assim como o desenho de estudo transversal e tamanho da amostra pequeno pode ter impedido a identificação de demais associações entre variáveis. Além disso, estudos que envolveram questionários contaram com a capacidade do indivíduo de interpretar as perguntas e fornecer respostas precisas, mas as respostas não foram verificadas objetivamente e, portanto, algumas relações causais podem ter sido perdidas ${ }^{(16)}$. Do mesmo modo, os níveis autorreferidos sobre os parâmetros de saúde mental nem sempre estão alinhados com a avaliação de profissionais que cuidam da saúde mental, os participantes podem ter fornecido respostas socialmente aceitas de acordo com as informações de saúde recebidas ${ }^{(6)}$.

\section{Recomendações para futuras pesquisas}

Estudos de coorte adicionais com mais amostras devem ser realizados e métodos não subjetivos devem ser utilizados. Por exemplo, o sono pode ser medido objetivamente por polissonografia e os níveis de estresse podem ser identificados usando medidas objetivas dos níveis séricos de cortisol ${ }^{(16)}$. Também seria ideal conduzir um estudo prospectivo, de acompanhamento do mesmo grupo de participantes investigados por um tempo maior. Isso se mostra importante, uma vez que os estudos que compuseram esta revisão analisaram os impactos da COVID-19 na saúde mental da população chinesa no início da epidemia, entre janeiro e fevereiro, quando o vírus começou a se espalhar e as cidades iniciaram restrições de movimento e quarentena. Contudo, medidas de contenção, como a supressão, iniciaram no país ao final do mês de janeiro, o que determinou uma paralisação em todo país, exigindo que todo residente chinês ficasse em casa até que a curva da doença fosse achatada. Neste sentido, o acompanhamento prospectivo dos indivíduos desde o início da epidemia até os dias atuais pode fornecer descobertas concretas para apoiar a necessidade de uma iniciativa de saúde pública direcionada ${ }^{(6)}$.

Também se recomenda que futuras pesquisas avaliem a eficácia de intervenções baseadas na medicina tradicional chinesa - como técnicas de acupressão e liberdade emocional - como forma de melhorar a saúde mental da população durante momentos de epidemia ${ }^{(6)}$.

\section{CONCLUSÃO}

Os estudos que compuseram a presente revisão avaliaram os impactos da COVID-19 na saúde mental da população chinesa no início da epidemia e evidenciaram a presença de indicadores emocionais negativos, como: a ansiedade, depressão, estresse, indignação, diminuição da felicidade, aumento da sensação de risco social e diminuição na satisfação com a vida, redução da qualidade do sono e níveis baixos de capital social.

Apesar dos atuais estudos serem realizados apenas em um país, a China, os resultados podem auxiliar no entendi- 
mento das condições da saúde mental daquela população e servir de exemplo para a realização de pesquisas em outros países, que devem avaliar a saúde mental da sua própria população com o intuito de direcionar iniciativas de saúde pública. Os resultados da presente pesquisa também mostram recomendações oportunas para gestores de políticas públicas, profissionais da saúde e à população para o manejo da saúde mental em situações emergenciais de saúde pública.

\section{AGRADECIMENTOS}

Este estudo foi financiado em parte pela Coordenação de Aperfeiçoamento de Pessoal de Nivel Superior - Brasil (CAPES) - Código Financeiro 001.

\section{REFERÊNCIAS}

1. Shereen MA, Khan S, Kazmi A, Bashir N, Siddique R. COVID-19 infection: Origin, transmission, and characteristics of human coronaviruses. Journal of Advanced Research [Internet]. 2020 Jul; 24:91-8. DOI: 10.1016/j.jare.2020.03.005.

2. Rothan HA, Byrareddy SN. The epidemiology and pathogenesis of coronavirus disease (COVID-19) outbreak. Journal of Autoimmunity [Internet]. 2020 May; 102433. DOI: 10.1016/j. jaut.2020.102433.

3. Wang $Y$, Di $Y$, Ye J, Wei W. Study on the public psychological states and its related factors during the outbreak of coronavirus disease 2019 (COVID-19) in some regions of China. Psychology, Health \& Medicine [Internet]. 2020 Mar. DOI: 10.1080/13548506.2020.1746817.

4. Qiu J, Shen B, Zhao M, et al. A nationwide survey of psychological distress among Chinese people in the COVID-19 epidemic: implications and policy recommendations. General Psychiatry [Internet]. 2020 Mar; 33(2). DOI: 10.1136/gpsych-2020-100213.

5. Asmundson GJG, Taylor S.How health anxiety influences responses to viral outbreaks like COVID-19: What all decision-makers, health authorities, and health care professionals need to know. Journal of Anxiety Disorders [Internet]. 2020 Mar; 71. DOI: 10.1016/j.janxdis.2020.102211.

6. Wang C, Pan R, Wan X, Tan Y, Xu L, Ho CS, Ho RC. Immediate Psychological Responses and Associated Factors during the Initial Stage of the 2019 Coronavirus Disease (COVID-19) Epidemic among the General Population in China. International Journal of Environmental Research and Public Health [Internet]. 2020 Mar; 17(5). DOI: 10.3390/ijerph17051729.

7. Xiao C. A Novel Approach of Consultation on 2019 Novel Coronavirus (COVID-19)-Related Psychological and Mental Problems: Structured Letter Therapy. Psychiatry Investigation [Internet]. 2020; 17(2):175-176. DOI: 10.30773/pi.2020.0047.

8. Park SC, Park YC. Mental Health Care Measures in Response to the 2019 Novel Coronavirus Outbreak in Korea. Psychiatry Investigation [Internet]. 2020 Feb; 17(2):85-6. DOI: 10.30773/ pi.2020.0058.

9. Ho CS, Chee CY, Ho RC. Mental Health Strategies to Combat the Psychological Impact of COVID-19 Beyond Paranoia and Panic. Annals of the Academy of Medicine, Singapore. 2020 Mar; 49(3):1-3.

10. Graeff FG. Ansiedade, pânico e o eixo hipotálamo-pituitária-adrenal. Revista Brasileira de Psiquiatria [Internet]. 2007; 29(1) s3-s6. DOI: 10.1590/S1516-44462007000500002.

11. Cirqueira FN, Coimbra GJ, Silveira AG. Influência do Estres- se Sobre o Sistema Imunológico - Example of Construction and Formatting of a Scientific Article [Internet]. ICESP, 2020 [acesso em 20 abr 2020]. Disponível em: http://nippromove.hospedagemdesites.ws/anais_simposio/arquivos_up/documentos/artigos/844c84423cfcd7e05d2720770d2ee271.pdf.

12. Marques-Deak A, Sternberg E. Psiconeuroimunologia: a relação entre o sistema nervoso central e o sistema imunológico. Revista Brasileira de Psiquiatria [Internet]. 2004; 26(3):143-144. DOI: https://doi.org/10.1590/S1516-44462004000300002.

13. Organização Mundial da Saúde. In: OMS [Internet]. [S. I.], 2020 [acesso em 03 abr 2020]. Disponivel em: https://www.who.int/ eportuguese/countries/bra/pt/.

14. Phillips B, Ball C, Sackett D, Badenoch D, Straus S, Haynes B, Dawes M. Oxford Centre for Evidence-Based Medicine - Levels of Evidence [Internet]. CEBM, 2009 [acesso em 20 abr 2020]. Disponivel em: https://www.cebm.net/2009/06/oxford-centre-evidence-based-medicine-levels-evidence-march-2009/.

15. Pedrosa KKA, Oliveira ICM, Feijão AR, Machado RC. Enfermagem Baseada em Evidências: Caracterização dos Estudos no Brasil. Cogitare Enfermagem [Internet]. 2015 [acesso em 20 abr 2020]; 20:733-741. Disponível em: http://docs.bvsalud.org/biblioref/2016/08/1128/40768-166899-1-pb.pdf.

16. Xiao $\mathrm{H}$, Zhang $Y$, Kong D, Li S, Yang N. The Effects of Social Support on Sleep Quality of Medical Staff Treating Patients with Coronavirus Disease 2019 (COVID-19) in January and February 2020 in China. Medical Science Monitor [Internet]. 2020 Mar; 26. DOI: 10.12659.

17. Li S, Wang Y, Xue J, Zhao N, Zhu T. The Impact of COVID-19 Epidemic Declaration on Psychological Consequences: A Study on Active Weibo Users. International Journal of Environmental Research and Public Health [Internet]. 2020 Mar; 17. DOI:10.3390/ ijerph17062032.

18. Grace Y. Lim, Wilson W. Tam, Yanxia Lu, Cyrus S. Ho, Melvyn W. Zhang \& Roger C. Ho Prevalence of Depression in the Community from 30 Countries between 1994 and 2014 Sci. Rep. 2018; 8:2861.

19. Portes A. Social capital: Its origin and application in modern sociology. Annu Rev Soc. 1998; 24(1):1-24.

20. Utz S, Breuer J. The relationship between use of social network sites, online social support, and wellbeing: results from a six-wave longitudinal study. J Media Psychol. 2017; 29(3):115-25.

21. Mortensen CR, Becker DV, Ackerman JM, Neuberg SL, Kenrick DT. Infection breeds reticence: The e ects of disease salience on self-perceptions of personality and behavioral avoidance tendencies. Psychol. Sci. 2010; 21:440-447. 